\title{
Progress, Challenges and Lessons Learned in the Construction of Wendelstein 7-X
}

\author{
Torsten Bräuer, Thomas Klinger, Hans-Stephan Bosch and the W7-X Team
}

\begin{abstract}
This paper reports on experiences gained during the construction of the large, superconducting, optimized stellarator device Wendelstein 7-X. The goal of the project is to demonstrate the fusion reactor potential of optimized stellarators and to operate for the first time fusion-relevant plasmas under full steady-state conditions. In addition the validity of the engineering concept is to be proven under operating conditions. The actual construction of the device is now running since more than ten years and will require about three further years of assembly and integration. The current technical status of Wendelstein $7-\mathrm{X}$ is reviewed, the major construction challenges are highlighted, and conclusions are drawn from past experiences that may also be useful for other large-scale science projects.
\end{abstract}

Keywords-components; Stellarator, Wendelstein 7-X, assembly, component alignment, metrology, mock up

\section{Introduction}

THE Wendelstein 7-X device (W7-X) is a superconducting stellarator with optimized magnetic field geometry and a modular coil concept. It will be the largest optimized stellarator in the world with an average major radius of $5.5 \mathrm{~m}$, an average minor plasma radius of $0.53 \mathrm{~m}$ and a total weight of $725 \mathrm{t}$ [1]. It is designed to operate in steady-state mode ( $30 \mathrm{~min}$ discharges) at reactor relevant plasma parameters [2]. The device has a five- periodic magnetic field symmetry and thus comprises five nearly identical magnet modules. A schematic drawing is shown in Fig. 1. Each module consists of two flipsymmetric half modules with five different non-planar and two different planar coils each. Each of the seven coil types can be independently energized to provide a wide range of operational flexibility. In addition there are two control coils and one trim coil per module for further experimental flexibility and compensation purposes. The 50 non-planar and 20 planar coils are superconducting (liquid $\mathrm{He}$ cooled $\mathrm{NbTi}$ cable-in-conduit with $18.2 \mathrm{kA}$ and $16 \mathrm{kA}$ nominal current, respectively) and are designed to produce a magnetic induction of up to $3 \mathrm{~T}$ on the magnetic axis.

\section{Manuscript received August 1, 2011}

The authors are with the Max-Planck-Institut fuer Plasmaphysik, Euratom Association, D-17491 Greifswald, Germany (email: torsten.braeuer@ipp.mpg.de; thomas.klinger@ipp.mpg.de, hansstephan.bosch@ipp.mpg.de)
To cope with the magnetic forces, planar and non-planar coils are fastened to a cast steel central support structure by a bolted connection with possible gap opening at the flange. The narrow support elements and the lateral support elements mechanically connect adjacent coil casings on the high field and on the low field side of the machine, respectively. The narrow support elements are sliding contacts, while the lateral support elements are welded connections with the exception of the (bolted) inter-module support elements.

The cold mass (coils and support system) is $425 \mathrm{t}$ in total and is cooled with liquid Helium to $3.4 \mathrm{~K}$. All cold components are in the cryostat volume which is formed by the plasma vessel, the outer vessel and the ports. 254 ports are designed for plasma diagnostics, heating, water supply of invessel components, and vacuum pump-down. The ports are welded on corresponding openings in the plasma vessel and domes on the outer vessel. They are equipped with bellows to compensate for deformations and displacements between plasma vessel and outer vessel. All vessels and ports surfaces are insulated by a multilayer insulation (Al coated Kapton ${ }^{\circledR}$ foil) which is completely covered with a thermal shield (glass fibre composite or brass with liquid Nitrogen cooling $80 \mathrm{~K}$ ) to minimize the heat load of the cryogenic components.

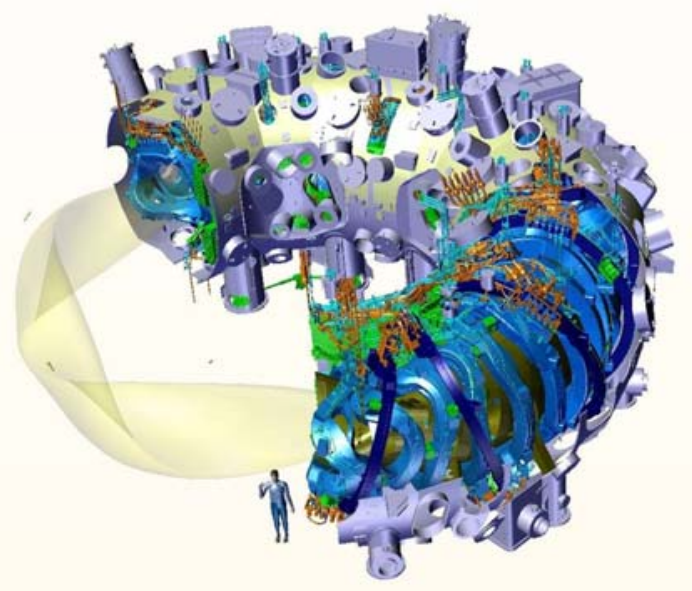

Fig.1: Schematic drawing of the stellarator Wendelstein 7-X. Shown are the non-planar coils (blue), planar coils (dark blue) the plasma vessel (olive green), the outer vessel (light beige).

The in-vessel components comprise the divertor targets and baffles, vertical closures, wall panels and heat shields for wall protection. For each of the ten divertor modules, a control coil is foreseen for strike-point position control. All in-vessel components are pressure water-cooled. A complex cooling pipe system, sensors, cables, and a number of embedded 
diagnostics complete the in-vessel system.

\section{Status of components}

Nearly all components of the magnet system are manufactured and delivered on site. For the key components, the 50 non planar coils and 20 planar coils, delivery and testing in a cryogenic test facility took in total six years [3]. It is the philosophy of the project to test all cryogenic major parts before final acceptance. This includes geometrical measurements, leak tests, for the coils in addition high voltage and quench tests at cryogenic temperatures. The 10 central support structure segments - to form a central ring - and all inter-coil support and connection elements are completed, too. Only a few elements used for joining modules on the separation planes (shim plates between flanges and bolted lateral support elements between the non planar coils) are left to be manufactured according to the detailed geometry.

The plasma vessel is composed of ten half modules, which are divided into two sectors each. The manufacturing of all 20 sectors was already completed in 2005 . The installation of the thermal insulation of the plasma vessel is completed. The outer vessel is made of five modules, each divided into an upper and a lower shell. All modules are manufactured and on site. Fig. 2 shows a photo of one outer vessel module. Note that large number of openings and domes. The thermal insulation of the last module of the outer vessel is close to completion.

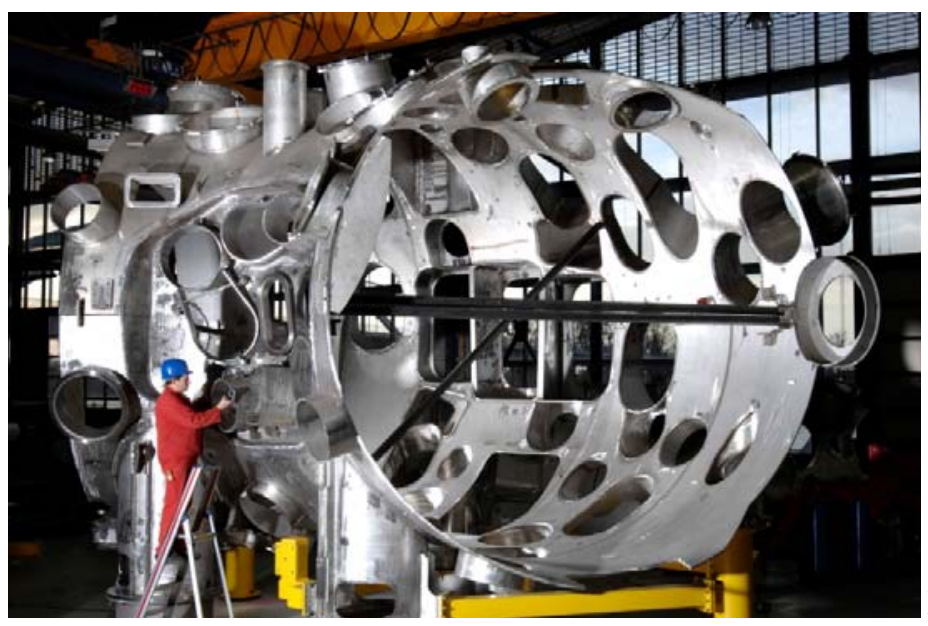

Fig. 2: Final inspection of one outer vessel module at the manufacturer site. Upper and lower shells are trial assembled.

As mentioned above, W7-X has a total number of 254 ports with a large variety of port geometries (about 100 different types). The port cross sections range between $100 \mathrm{~mm}$ in diameter (round cross section) and $400 \times 1000 \mathrm{~mm}^{2}$ (rectangular cross section). All ports were already delivered in 2007. The two special ports for neutral beam injection are currently reworked by the manufacturer to allow assembly within the small tolerances required. Dummies of this port type were manufactured to test the assembly procedures.
The cryo pipe system of W7-X distributes the liquid Helium within the cryostat. It starts at the Helium port on the outer vessel and ends in individual feeders of the cold components. Due to the highly constrained space available in the cryostat volume, the routing of the cryo pipes turned out to be a major challenge. This resulted in a pronounced 3Dgeometry and about 1000 different (low Cobalt content) stainless steel pipes were to be bent, orbital welded, geometrically checked, leak tested and finally delivered. About 700 stainless steel clamps with glass-fiber inlays were manufactured to support the pipes. All these parts are now manufactured and delivered on site. Fig. 3 shows a completed magnet module, highlighting the difficult space situation in the cryostat.

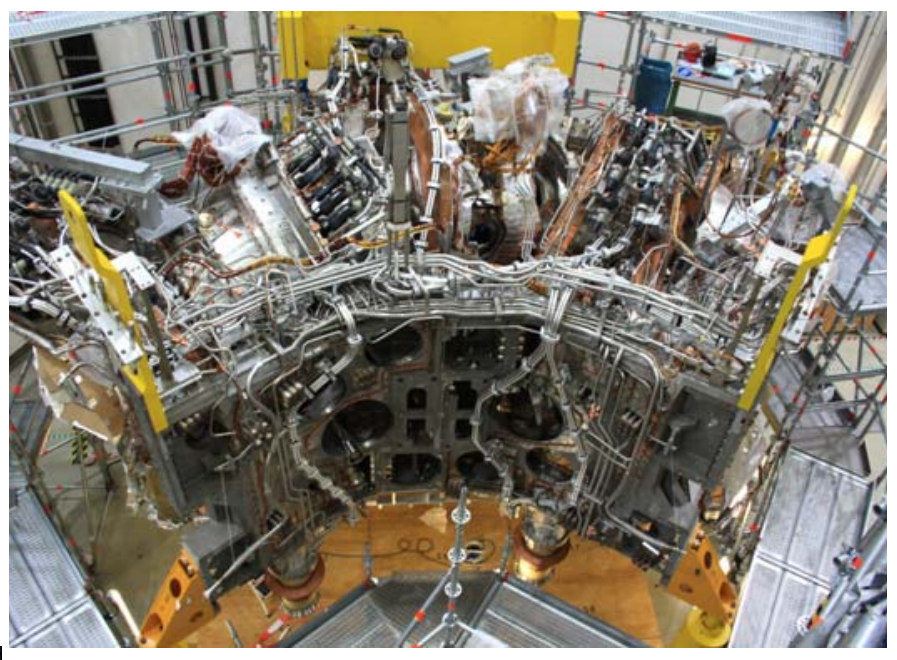

Fig. 3: View of a completed magnet module. In the foreground, mostly on and above the central support ring, the liquid Helium distribution system is seen, sharing the limited space with the superconducting bus bar system.

For the seven $20 \mathrm{kA}$ electric circuits that are connecting in series the superconducting coils of the same type, the same superconductor is used as for the coils. All 113 conductors (243 $\mathrm{NbTi}$ strands, Al cable in conduit cross section $\approx$ $16 \times 16 \mathrm{~mm}^{2}$ with a length of up to $15 \mathrm{~m}$ ) have been bent into the designed contour and delivered [4]. Also manufactured and delivered are the $\sim 400$ clamps and holder elements for the fixation of the superconductor.

The current leads are the electrical connections between the superconducting magnet system inside the cryostat and the power supplies outside the cryostat. They establish the transition between cryogenic and room temperature and between superconductivity and normal conductivity. For the magnet system's seven current circuits, in total 14 current leads are manufactured. For W7-X layout reasons, the warm end of the current lead is designed to be on the bottom ("upside down", i.e., against the convection direction). The prototypes have been developed at the Karlsruhe Institute of Technology [5] and have recently been successfully tested. The series production has started. Fig. 4 shows a photo of the current lead prototype. 


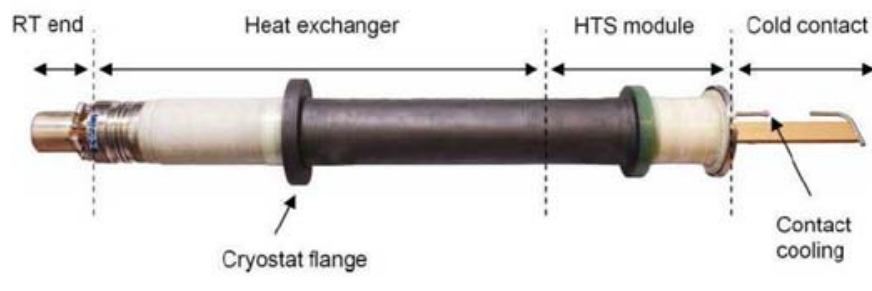

Fig. 4: Prototype of current lead. The design is qualified for assembly with the room temperature end at the bottom.

To fine tune the edge magnet field geometry and to further increase the flexibility of the magnet field structure, five normal conducting saddle coils are symmetrically installed on the outside of the cryostat (Fig. 5). These trim coils (one per module) have a size of about $3.6 \times 3.3 \mathrm{~m}^{2}$ (four coils type A) and $2.8 \times 2 \mathrm{~m}^{2}$ (one coil type $\mathrm{B}$ ), respectively. They are operated at about $2 \mathrm{kA}$ and water cooled. The design of the coils is fixed and the manufacturing will soon start. The coils are provided in-kind by the US fusion program in the framework of a cooperation program. Already started is the manufacturing of the coil support elements, which have to be fixed on the outer vessel.

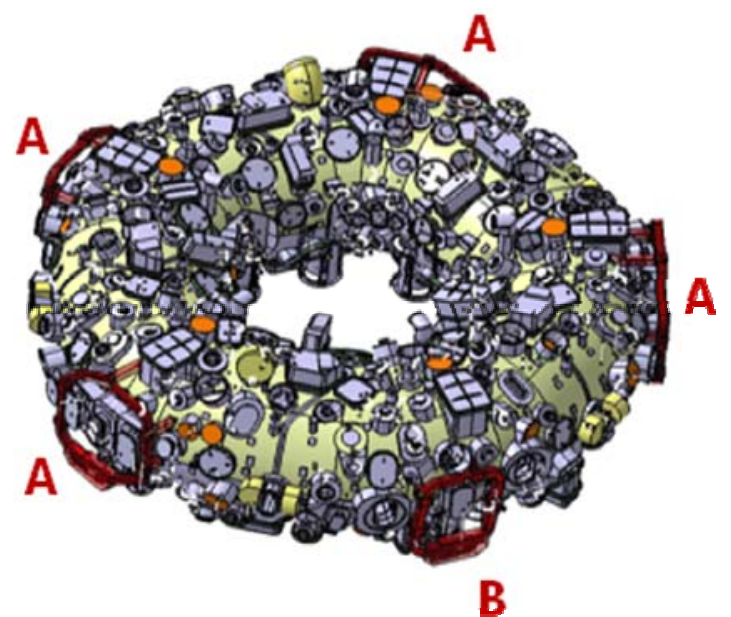

Fig. 5: Normal conducting trim coils - one saddle coil per module - used to fine tune the edge magnet field geometry.

The manufacturing of the in-vessel components is well advanced and many components were already delivered. The divertor target elements, water cooled steel panels, cooling water supply lines, and the control coils are mainly manufactured in industry (the latter already completed). Baffles, heat shields, cryo pumps, port protective liners, and the test divertor units are mainly manufactured in house. For a first operation phase with discharge duration $8-10 \mathrm{~s}$ at full heating power, inertially cooled test divertor units will be installed. They will be later replaced by the high heat flux divertor units (pressure water cooled) for achieving steadystate operation [6]. In the manufacturing process are still the heat protection shields (125 of 162 delivered), the test divertor units, the cooling circuit pipes (80 of 199 delivered), and the high heat flux target elements (about 100 of 890 delivered). The target elements of the high heat flux divertor consist of carbon fiber reinforced carbon (CFC), HIPed and electron beam welded to a $\mathrm{CuCrZr}$ heat sink. They are subject to a thorough testing program, including a high heat flux test with 100 cycles at $10 \mathrm{MW} / \mathrm{m}^{2}$ heat flux.

\section{Status of assembly}

\section{Component preparation and assembly tool development}

Since about one year, the project has fully moved from the component-dominated to the assembly-dominated phase [7]. The first assembly step is the preparation work on the components. The preparation work on coils, plasma vessel, and support structure segments is done. On the outer vessel shells, both the mechanical works and the installation of the thermal insulation run routinely. End of 2011, the last completed outer vessel is ready for final assembly.

The port preparation work runs routinely and according to schedule. Until 2013 port assembly determines the critical path of the device construction. To reduce the respective process time, the originally planned trial assembly of the ports was substituted by a metrology method for cutting contour and port length determination. This approach, based on the conversion of metrology data to a model based cut-contour, is consuming more metrology and design office resources but reduces considerably the overall process times due to better parallelization. As a consequence the port assembly is running according to schedule. The tools for port assembly as well as the respective welding procedures were systematically optimized to guarantee the required positioning accuracy $(+/-$ $3 \mathrm{~mm}$ at the dome end) while keeping the schedule. For the general assembly and welding procedures, in particular for the most complicated ports, dedicated simulation runs using port dummies were performed.

The concepts for the current leads assembly procedures are completed and are tested on a 1:1 mock-up. For the lifting of the long and sensitive current leads, a modified port assembly ramp is used.

The next challenge in the device assembly will be the integration of more than 2000 large, 3d-shaped in-vessel components [6]. Trial assemblies for the wall panels, heat shields, sweep coils, and cooling water pipes have been successfully conducted. Platforms, cranes for the handling of components, positioning tools were further developed and tested under realistic construction site conditions. A manipulator device for the precise 3d-positioning of supports and bolts in the interior of plasma vessel was put into operation and is intensively tested. Up to now, the required adjustment accuracy requirements could be achieved.

\section{Pre-assembly}

All five magnet modules have been completely assembled with some remaining (minor) works on the last one. Fig. 3 (above) shows the top view of a completed magnet module.

In the first pre-assembly step ten half modules were assembled, each consisting of five non planar coils, two planar coils, one support structure segment and two plasma vessel 
sectors. All ten half modules were successfully assembled and meet the quality requirements. In the second pre-assembly step, two half modules are bolted and welded together. In that assembly step, too, the high geometric accuracy requirements have been met: No part of a coil in all five modules deviates more than $3.2 \mathrm{~mm}$ from its nominal position. Due to that enough assembly tolerance remains to meet the estimated maximum worse case deviation of $6.5 \mathrm{~mm}$ of each point of a coil after final assembly of the torus.

Magnetic field calculations, based on the as-build positions of the coils, have shown that the resulting field perturbations can be accepted. To improve the magnet field quality further the calculated field errors are minimized by mathematical variation of position and orientation of the as-built modules and defining new co-ordinates for the modules used at the final adjustment. In total a sphere of up to $5 \mathrm{~mm}$ radius is foreseen for that optimization process but not fully used up to now. The other module components were assembled in the required geometrical tolerances, too. In a few cases, e.g. coil casings, mechanical rework was necessary to avoid collisions between cold components.

In parallel to the geometrical measurements, leak tests, high voltages tests and electrical checks of sensors have been performed. All weld seams of Helium pipes made on the construction site were leak tested after a thermal shock to $77 \mathrm{~K}$. The busbar joints were locally tested at room temperature. The high voltage tests were performed under Paschen conditions [8]. The first four modules passed all tests successfully and could be released for final assembly in torus hall. The test program on the last magnet module is completed until the last quarter of 2011.

\section{Final assembly}

After completed pre-assembly, the magnet modules are inserted into the lower shell of the outer vessel and positioned onto the machine base on their final positions.

The alignment of all modules has been accomplished within the given tolerances. The magnet modules are aligned to their optimized co-ordinates (see above) within an adjustment residual lower than $1.5 \mathrm{~mm}$. After the alignment of each magnet module, the resulting magnetic field is recalculated and analyzed for field errors (in particular magnetic island formation). On the basis of these calculations it is expected that the resulting magnetic field will be fully satisfactory provided last magnet module is adjusted within the same alignment residuals as achieved for the first four modules.

The alignment of outer vessel shells and plasma vessels has been performed, too, within the specified margins of $1.5 \mathrm{~mm}$ and $2 \mathrm{~mm}$, respectively. During the alignment of the outer vessel, deformations of up to $14 \mathrm{~mm}$ occurred and were considered acceptable. The deformations were mostly owing to weld shrinkage and release of death weight forces. Fig. 6 shows the positioning and alignment of an outer vessel upper shell.

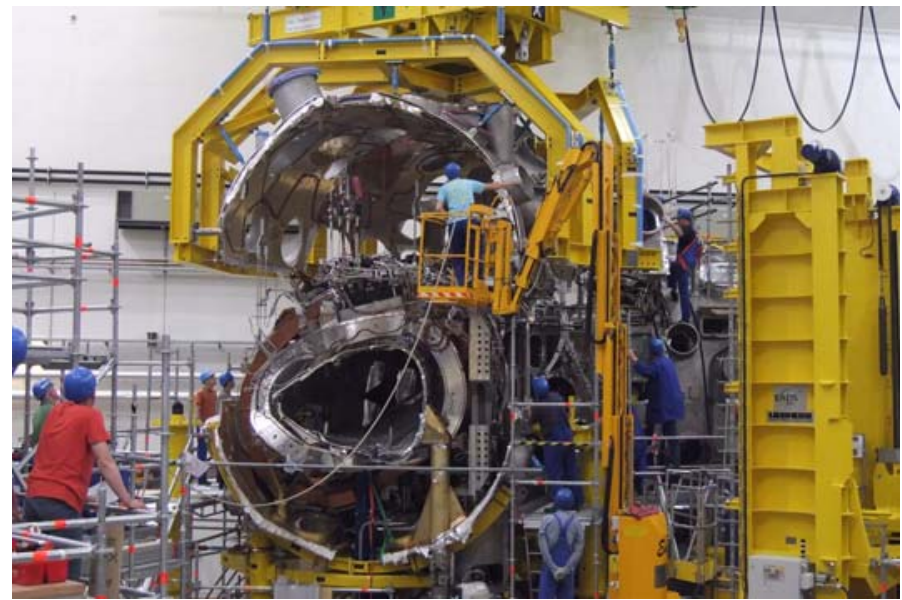

Fig. 6: Alignment of an outer vessel upper shell. After alignment upper and lower shell are welded.

After the two outer vessel shells of a module have been welded, port assembly starts. In the first out of five modules all ports have been installed, welded and leak tested. A few ports turned out to be a few mm out of tolerance and a port assembly improvement program has been launched. The improvement program is based on (a) permanent full metrology of the port position during welding, (b) optimized welding technology with intermediate $\mathrm{CO}_{2}$ cooling, (c) additional stops, tools and supports for dead weight compensation. In the second module, all ports are now installed, aligned and tack welded. The final welding has started and, owing to the above described improvements of the assembly and welding procedures, the accuracy of the port positions is now fully satisfactory. In the third and the fourth module, port installation and tack welding is in progress. Additional smaller improvements in the assembly technology and the introduction of metrology-guided tack welding have the alignment quality further improved. Overall, the port assembly is now running routinely, but more resources are consumed than originally assumed. To meet the schedule milestones, additional work force from industry was hired to cover the present work scheme with 88 hours per week. Fig. 7 shows the initial phase of the assembly of a complex shaped double port. 


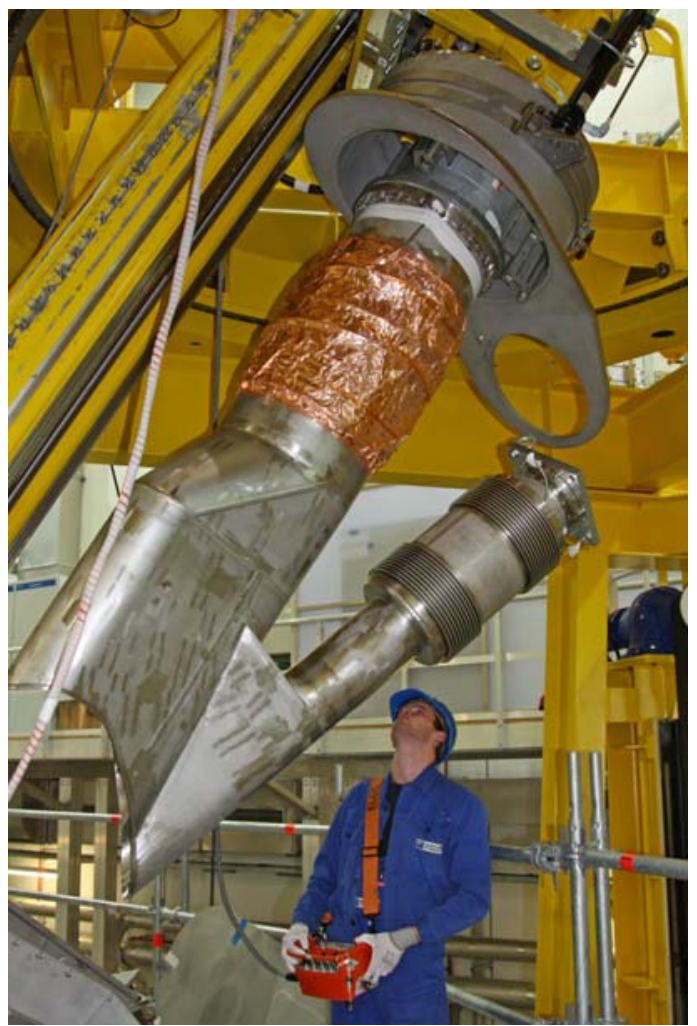

Fig. 7: Port assembly using the linear positioning ramp (yellow device in the top). The $3 \mathrm{~d}$ contour of the port (left hand side) is determined from $3 \mathrm{~d}$ laser scans and cutting in the port assembly preparation process.

In parallel to the port assembly, the final joining of the first two device modules has been started. For the central support structure of the magnet modules, 3d-shaped shims are manufactured to bridge the gap $(\approx 35 \mathrm{~mm})$. Each shim must be individually customized within an accuracy of better than $0.1 \mathrm{~mm}$, which is at the technical limits. The gap dimension is determined by using steel-templates and a co-ordinate measurement device. The works on the next two module separations will start soon.

\section{Challenges in the next assembly steps}

The port assembly, despite running smoothly, will remain a technical challenge until the very end because of the geometrical complexity of the ports and the complex alignment and welding process to the $3 \mathrm{~d}$-shaped plasma vessel. However, there are four other challenging working packages arising in the near future: (1) the joining of outer and plasma vessels, (2) the assembly of the components in plasma vessel, (3) the current lead assembly, and (4) periphery and diagnostics installation in the torus hall.

The prerequisite for the connection of outer and plasma vessels of neighboring modules is the completion of all mechanical work to connect the respective magnet modules. Two lateral supports to support the coils of adjacent modules are in the manufacturing and will have to be assembled. Each support element weights about $50 \mathrm{~kg}$ and has to be installed through a relatively narrow gap between the two outer vessel shells of the neighboring modules. For positioning and tightening of the bolts, one has to work in the interior of the module in a narrow assembly duct. The support elements are a complex, twisted wedge construction. They must fit into their counter parts on the coil housings with accuracy better than $0.05 \mathrm{~mm}$. To achieve that, the individual shape of the coil counter parts is scanned used a $3 \mathrm{~d}$ stripe projector method. The position of the two opposite counter parts is determined with photogrammetry. Both data sets together, scan and photogrammetry, form the base for the manufacturing of an aluminum dummy wedge. The dummy is trial installed and reworked until the remaining gap between dummy and the coil counter parts is smaller than $0.05 \mathrm{~mm}$. The aluminum dummy is the base for the consecutive manufacturing of the final support made from stainless steel EN 1.4429 (similar to AISI 316 LN).

The connection of outer and plasma vessels of the first and second module is in preparation and scheduled for end 2011. To cope with the weld shrinkage, the following procedure is applied: The vessels of both modules are separated by 4-6 $\mathrm{mm}$. One module (the first one) is fixed to the machine base. The second module is assumed to move perpendicular to the module separation plane by the pre-calculated weld shrinkage. The movement is monitored by metrology and the starting position for the welding process is determined for all vessels. The resulting clearance between each pair of vessel modules about $140 \mathrm{~mm}$ - is measured and individual 3d-shaped steel plates are pre-manufactured to fill the gap. During welding of the vessels, the vessel position is monitored by up to three simultaneously operating laser-trackers. The 3d-metrology data are available on-line and are used by the welding team to control the process and to initiate counter measures in case of strong deviations.

After the first two plasma vessels are connected and the few ports located on the module separation plane are installed, the assembly of in-vessel components starts. As a first step, the weld studs and supports are aligned and welded on the plasma vessel surface. They are used for installing the plasma facing components and various kinds of pipes.

The first qualification tests of the manipulator (see above) have shown that the required positioning accuracy of $1.5 \mathrm{~mm}$ for studs and supports can be achieved. In the global coordinate system the alignment tolerances of plasma facing components - divertor targets, baffles, panels, and heat shields - are of the same order than the tolerances of their support elements. To avoid steps and leading edges between neighboring components, the local alignment tolerances are smaller and may reach a few $1 / 10 \mathrm{~mm}$.

Assembly personnel and all components enter the plasma vessel via man ports (cross section 400 x $1000 \mathrm{~mm}^{2}$ ) after passing an airlock. For lifting and positioning of components a rail system hanging on the top of the vessel was designed and successfully tested. For the majority of the in-vessel component assembly metrology support is required. The use of metrology equipment is hampered by tight space conditions in the vessel. As for the water distributions system, even by using 
preassembled manifolds, a large number of - mostly orbital welds must be done inside the vessel. Each weld seam on the cooling pipe system has to be leak tested at a temperature of $430 \mathrm{~K}$. Fig. 8 shows the trial assembly of an in-vessel cooling pipe manifold.

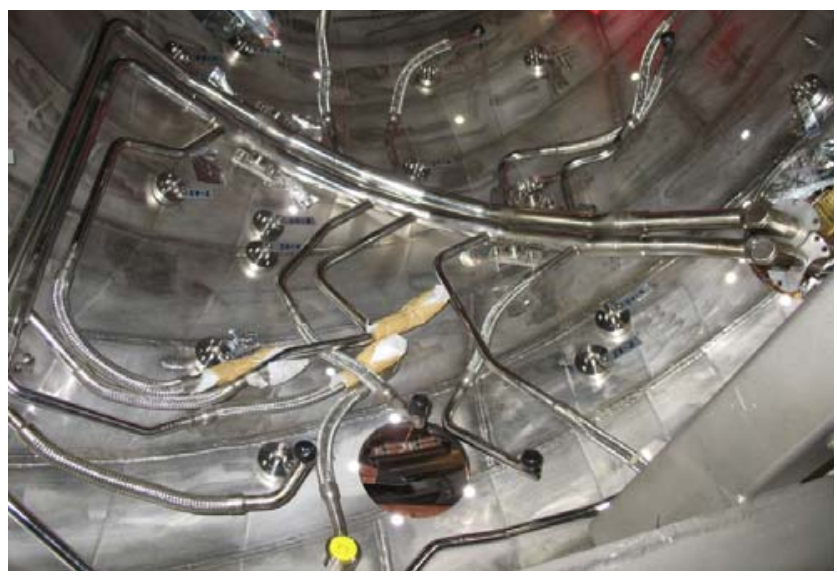

Fig. 8: Complex pipe work inside plasma vessel. Preassembled, leak checked manifolds are used but many weld seams still have to be done inside the plasma vessel.

In addition to the above described technical challenges, the assembly logistics will become very demanding. The assembly of in-vessel components will be done on all modules in parallel by several assembly teams. This requires a complex and highly flexible resource planning. The presently established two shift system with up to $88 \mathrm{~h} /$ week working time is foreseen for the future as well. From mid 2013 onwards, this work will determine the critical path of the overall construction project.

As mentioned above, the assembly concept for the current leads was carefully planned and verified in various assembly trials on 1:1 mock-ups. The alignment tools are designed and tested as well, among them a modified assembly ramp, which is already in use for the port assembly.

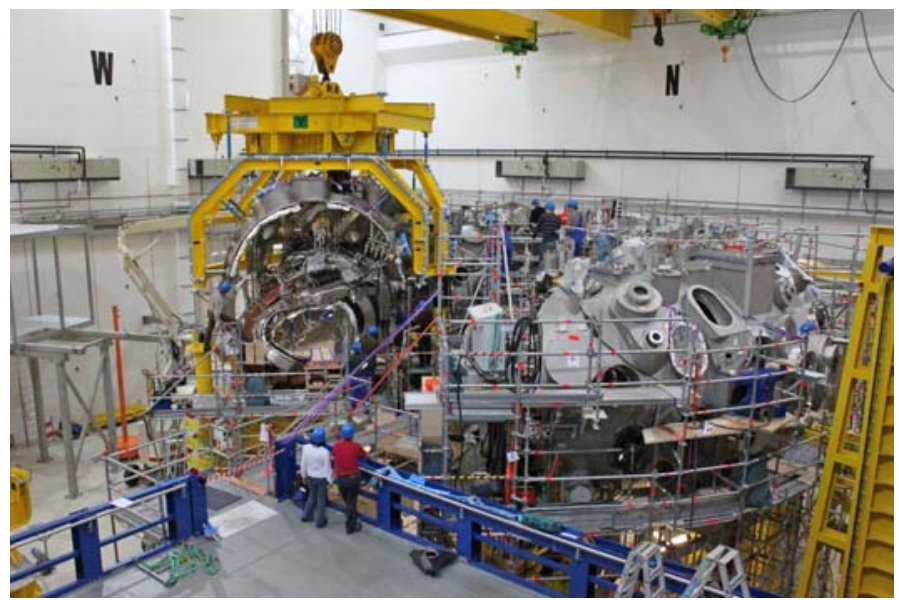

Fig. 9: View into the torus hall in April 2011. Four out of five modules are positioned on the machine base. The upper shell of the outer vessel is being assembled on the fourth module.

The installation of the periphery has started at the first module. The second stage of the cooling water pipe system in the torus hall has been launched and the first lot of cable trays has been mounted. The detailed planning for the periphery and torus hall layout (cable trays, cooling pipes, liquid gas supply, vacuum lines, platforms, diagnostics, heating systems, supply systems) is running and will be completed until fall 2011. Fig. 9 shows a recent view into the torus hall of Wendelstein 7-X.

\section{Lessons learned}

The stellarator is by nature a more complex device than a tokamak. However, the main issues that have led to serious difficulties in the project development seem to hold for any state-of-the-art fusion and non-fusion projects. There were a variety of technical and organizational problems that have led to both cost overruns and schedule slippages (e.g. for the magnets cf. Ref. [8]). These issues were mostly resolved andafter a thorough revision of the planning base in 2007 - the project is now running since four years in schedule and in budget. Nevertheless, it is worth summarizing in brief the lessons learned from the construction of Wendelstein 7-X.

1. A competent project team must be set up prior to design completion, component specification and start of procurement. Any lack in the work done at this stage will create dramatically higher efforts, in cost and time, later in the project.

2. Recruitment and head-hunting must be taken extremely serious. Where the know-how is not available, strong external institutional partners must be found and deeply integrated into the project. During team formation, the human factor must be seriously taken into account.

3. There must be 5-10 persons in the project that know the machine from $a-z$. Experience with large construction projects is invaluable. Clear project structures and responsibilities are mandatory. Matrix structures should be avoided where ever possible.

4. The project management must be put into the situation to identify risks precisely and timely enough to react with reasonable countermeasures. High-level management tools must be implemented and accepted on the working level. Formal procedures and written documents are unavoidable, but must be organized as pragmatic as possible.

5. A lack of generous margins, clearances and reasonable tolerance levels implies uncontrolled and unnecessary increase of complexity and frequent changes. This has a strong impact on time, costs and man-power demands and potentially sours the relationship to funding bodies.

6. Major components must be thoroughly tested and qualified prior to tender action. The manufacturing process must be accompanied by a dedicated in-house test program based on manufacturing samples and mockups. Quality management must be involved in 
each single step, starting with the component design. Experienced inspectors must follow up manufacturing in detail.

7. Development and manufacturing risks must be taken to a large extent by the project. Industry cannot do that or will charge the project to cover unexpected costs, even far beyond the contract. The only solution is to solve suddenly arising technical problems stepby-step together with industry.

8. The construction of first-of-a-kind devices requires specialized in-house knowledge and specialized, qualified industrial suppliers with sufficient capacity available. Monopoly situations often cannot be avoided and must be taken into account in design, planning and contract management. Loss of expertise or shifts of priorities in industrial partners may happen on short time-scale and must be permanently checked on the manufacturing site.

\section{Summary}

The fabrication of the major components of the Wendelstein 7-X device has been finished, in particular the manufacture of superconducting coils, plasma and outer vessel, central support structure and ports. For the remaining smaller components, mainly the thermal insulation, the components in plasma vessel, the current leads and the periphery, design and manufacturing runs according to schedule. Four out of five magnet modules have been completely pre-assembled, the fifth one is completed soon. The four completed magnet modules have been inserted into the outer vessel modules and moved to their final position on the machine base.

The analysis of the achieved assembly accuracy has shown that of the expected magnet field geometry based on actual module positions show that the required field quality can be achieved. The port assembly on the first module has been completed. In a few cases the alignment accuracy was not satisfactory and counter measures were necessary. After systematic optimization of the port assembly and welding procedures and use of additional metrology, port assembly now runs smoothly. In addition to port assembly, there are four challenging working packages ahead: the connection of plasma and outer vessels of adjacent modules, the assembly of the large number of in-vessel components, the assembly of the current leads, and installation of periphery including the overall torus hall layout. Trials, partly on 1:1 mock-ups, have been done to mitigate the risks in the most critical assembly steps.

The construction project Wendelstein $7-\mathrm{X}$ is now well on track and both schedule and budget are stable since more than four years. There were, however, serious technical and managerial crisis and a number of "lessons learned" were briefly summarized at the end of the paper. Generally speaking, the construction of a superconducting fusion device has very often a first-of-a-kind character and requires a tailored approach, taking into account boundary conditions arising from the available budget, formal rules, the political framework, and the specific situation of all partners involved. Nevertheless some of the observations made during the evolution of the construction project might be useful for other big science projects, too.

\section{REFERENCES}

[1] G. Grieger et. al., "Physics optimization of stellarators", Physics of Plasmas 4 (1992) 2081-2091.

[2] H.-S. Bosch et al., Construction of Wendelstein 7-X - engineering a steady state stellarator, IEEE Transactions on Plasma Science 38 (2010) 265-273

[3] K. Risse et al., "Design, Tests, and Repair Procedures for the Electrical Insulation of the Superconducting W7-X magnets", IEEE Transactions on Applied Superconductivity 20 (2010) 447-450.

[4] O. Neubauer et al., "The busbar system for Wendelstein 7-X prepared for assembly and operational loads", Fusion Engineering and Design 84 (2009) 1416-1419.

[5] R. Heller et al., "Test results of the high temperature superconductor prototype current leads for Wendelstein 7-X", IEEE Transactions on Applied Superconductivity 21 (2011) 1062-1065.

[6] R. Stadler et al. "The in-vessel components of the experiment Wendelstein 7-X", Fusion Engineering and Design 84 (2009) 305-308.

[7] L.Wegener, "Status of Wendelstein 7-X construction", Fusion Engineering and Design 84 (2009) 106-112.

[8] C. Sborchia, "The manufacture of the W7-X supercoducting magnet system as relevant experience for the construction of next fusion devices", IEEE Transactions on Applied Superconductivity 17 (2007) 1334-1337.

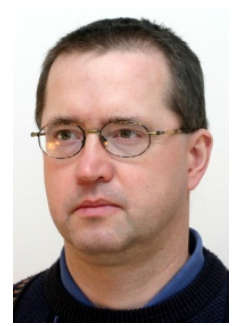

Torsten Braeuer received the Dipl.-Phys. degree and the Dr. rer. nat. degree from the Ernst-Moritz Arndt University, Greifswald, in 1991 and 1997, respectively.

$\mathrm{He}$ is currently head of the Metrology group of the Wendelstein $7-\mathrm{X}$ project at Max-Planck-Institut für Plasmaphysik, Greifswald. His research interests are Laser based geometrical measurements and plasma diagnostics. 


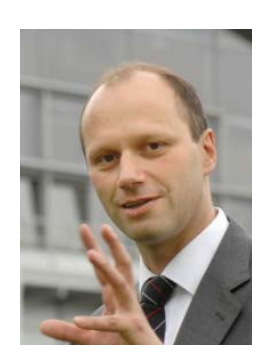

Thomas Klinger, born in 1965, studied physics at the University of Kiel, Germany. After a research period in France he took his $\mathrm{PhD}$ in 1994 with a thesis on gas discharge physics. As a research assistant at Kiel, he was working on drift wave turbulence and nonlinear plasma structures. After being as a visiting scientist with the Alfvén Laboratory in Stockholm, Centre de Physique Théorique in Marseille and Max-Planck-Institute of Plasma Physics in Garching he received his habilitation in 1998. In 1999 he was appointed as Professor of Experimental Physics at Ernst-Moritz Arndt University in Greifswald, where he has headed the Institute of Physics as Managing Director from 2000 till 2001. Since April 2001 he is Scientific Member of the Max-Planck Society and Director at the Max-PlanckInstitute of Plasma Physics (IPP), Greifswald site. Since 2005 he is member of the IPP executive board and Scientific Director of the stellarator project Wendelstein 7-X.

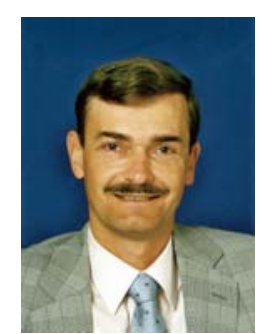

Hans-Stephan Bosch received the Dipl.Phys. degree from Ludwigs- Maximilians University Munich, Germany, in 1983, the Dr. rer. nat. degree from the Technical University, Munich, in 1986, and the Habilitation degree from Humboldt University, Berlin, Germany, in 2000.

$\mathrm{He}$ is a Lecturer with Greifswald University, Greifswald, Germany, since 2008. He is currently the Associate Director Coordination of the Wendelstein 7-X project at Max-Planck-Institut für Plasmaphysik, Greifswald. His research interests are plasma edge and divertor physics. 